\title{
The Definition and Measurement of the Topological Entropy per Unit Volume in Parabolic PDE's
}

\author{
P. Collet ${ }^{1}$ and J.-P. Eckmann ${ }^{2,3}$ \\ ${ }^{1}$ Centre de Physique Théorique, Laboratoire CNRS UMR 7644, Ecole Polytechnique, F-91128 Palaiseau Cedex, France \\ ${ }^{2}$ Dépt. de Physique Théorique, Université de Genève, CH-1211 Genève 4, Switzerland \\ ${ }^{3}$ Section de Mathématiques, Université de Genève, CH-1211 Genève 4, Switzerland
}

\begin{abstract}
We define the topological entropy per unit volume in parabolic PDE's such as the complex GinzburgLandau equation, and show that it exists, and is bounded by the upper Hausdorff dimension times the maximal expansion rate. We then give a constructive implementation of a bound on the inertial range of such equations. Using this bound, we are able to propose a finite sampling algorithm which allows (in principle) to measure this entropy from experimental data.
\end{abstract}

\section{Introduction}

In this paper, we shall deal with a general reaction-diffusion equation, and we have in mind an $N$-component system in $\mathbf{R}^{d}$ which is of the form

$$
\partial_{t} u_{i}(x, t)=\sum_{j=1}^{N} d_{i j} \Delta u_{j}+F_{i}\left(u_{1}, \ldots, u_{N}\right), \quad i=1, \ldots, N
$$

where all quantities are real. For example, the complex Ginzburg-Landau equation (CGL) is

$$
\partial_{t} v(x, t)=(1+i \alpha) v^{\prime \prime}(x, t)+v(x, t)-(1+i \beta) v(x, t)|v(x, t)|^{2},
$$

which clearly can be brought to the form of Eq.(1.1) by writing equations for the 2 components $u_{1}=\operatorname{Re} v, u_{2}=\operatorname{Im} v$. We shall write Eq.(1.1) short as

$$
\partial_{t} u=D \Delta u+F(u)
$$


We state now our assumptions on $D$ and $F$. We let $D_{\text {sym }}=\left(D+D^{\mathrm{T}}\right) / 2$ denote the symmetric part of $D$, and we assume that the matrix $D_{\text {sym }}$ has spectrum $\left\{\nu_{i}\right\}_{i=1, \ldots, N}$ in the open right half-line. We next define

$$
\nu_{*} \equiv \min _{i=1, \ldots, N} \nu_{i}, \quad D_{*} \equiv\|D\|,
$$

where $\|D\|$ is the norm of the matrix $D$ as a linear map from $\mathbf{R}^{N}$ (equipped with the $l^{2}$ norm) to itself. Note that $\nu_{*}$ is the minimal dissipation rate in Eq.(1.1). Our assumptions on $F$ are somewhat vague, but they are intended to cover a large variety of possible applications. We first assume that $F$ is "globally stabilizing" in the sense that there is a constant $Q_{*}$ such that for any initial condition $u(x, 0)$ which is bounded in $\mathrm{L}^{\infty}$ there is a $t<\infty$ such that $\left\|u\left(\cdot, t^{\prime}\right)\right\|_{\infty} \leq Q_{*} / 2$, for all $t^{\prime}>t$. (The factor $\frac{1}{2}$ is convenient for later use.) In this sense, $Q_{*}$ is the radius of a globally invariant set (usually this will be an attracting set). ${ }^{1}$ In the case of the real GinzburgLandau equation (Eq.(1.2) with $\alpha=\beta=0$ ), one has $Q_{*} / 2=1$ and for the CGL it has been shown in [C,GV] that $Q_{*}<\infty$ when $d=1$ or $d=2$, and also in dimension $d=3$ for some nontrivial parameter range of $\alpha$ and $\beta$. For many other equations one can derive similar bounds using the localization techniques of [CE1]. Since this is not the central issue of our paper, we shall just assume that

$$
\left\|u_{t}\right\|_{\infty} \leq Q_{*} / 2
$$

for all $t$. Here, and in the sequel $u_{t}(x)=u(x, t)$. Our last general assumption is a bound on the maximal local expansion rate. Consider two solutions $u$ and $v$ with $\left\|u_{t}\right\|_{\infty}$ and $\left\|v_{t}\right\|_{\infty}$ bounded by $Q_{*}$ for all $t \geq 0$. (This is no loss of generality if we consider later functions in the "global attractor" $\mathcal{A}$.) We define $w=u-v$. Then, we assume that the non-linearity $F$ is such that $w$ satisfies an equation of the form

$$
\partial_{t} w(x, t)=D \Delta w(x, t)+M(x, t) w(x, t)
$$

where the matrix $M$ has a norm (as a map from $\mathbf{R}^{N}$ to itself) bounded by

$$
\|M(x, t)\| \leq M_{*},
$$

for all $x$ and $t$. If $F$ is a polynomial, such a bound will follow automatically from the bound of Eq.(1.4). For example, for the CGL, written in complex notation, we have

$$
\partial_{t} w=(1+i \alpha) \Delta w+(1+(1+i \beta)(u \bar{v}+v \bar{v})) w+\left((1+i \beta) u^{2}\right) \bar{w},
$$

so that in this case $M$ is a $2 \times 2$ matrix whose norm is bounded by

$$
\|M\| \leq 1+3(1+|\beta|) Q_{*}^{2} / 4
$$

\footnotetext{
${ }^{1}$ We shall try to stick to the following notation: Quantities with a $*$ as an index depend on the parameters of the Eq.(1.1), and the constants $C_{0}, C_{1}, \ldots$ do not depend on them.
} 
Again, many other examples can be handled in this manner and are left to the imagination of the reader.

Our study of the topological entropy is based on a detailed analysis of the Eq.(1.5), and in particular on the control of information which is localized in space, in the conjugate momentum, and in time. The localization in space and time has been developed earlier [CE2] and used to prove the existence of the $\varepsilon$-entropy per unit volume of Kolmogorov and Tikhomirov [KT] for systems such as CGL. Here, we use these estimates to show the existence of the topological entropy per unit volume. We then improve the bounds to localize at high frequencies, where the flow defined by Eq.(1.5) will be seen to be essentially a contraction. Using this information, we shall then show that the topological entropy per unit volume can be measured in terms of a discrete sampling of the signal $u$ in space and time. The amount of data needed for such an enterprise is, however, quite prohibitive [ER2] if any reasonable precision is to be attained. But this is probably unavoidable. For a similar study in finite volume, see [CJT].

We end this introduction by explaining in more detail how the various physical scales interact as we bound $w$, since this should be useful to prove further results for dissipative systems in unbounded domains. We wish to argue in "dimensionally correct units" so that $w$ has the dimension of the observed fields (e.g., a temperature), $[M] \sim[t]^{-1}$ and $[D] \sim\left[\ell^{2} t^{-1}\right]$, where $t$ is time and $\ell$ is length. In the long wavelength limit, diffusion is inactive, and the growth of $w$ is dominated by $M$. Given the a priori bound $M_{*}$ on $M$, we shall fix the unit of time to

$$
\tau_{*} \equiv \frac{1}{M_{*}} .
$$

Recall that $D_{*}$ is the norm of $D$ and the (dimensionless) quotient $D_{*} / \nu_{*}$ compares essentially the strongest to the weakest dissipation rates. The time $\tau_{*}$ is the time in which errors can grow at most by a bounded factor, which depends on $D_{*} / \nu_{*}$, and we shall see that $1 / \tau_{*}$ is also the natural sampling rate for the determination of the entropy. The dissipative range of the equation (1.5) is given by those $k$-values for which dissipation is guaranteed to exceed the growth, i.e., for $\nu_{*} k^{2} \geq M_{*}$. Hence we set the cut-off for the $k$-values to

$$
k_{*} \equiv\left(\frac{1}{\tau_{*} \nu_{*}}\right)^{1 / 2} \cdot F\left(D_{*} / \nu_{*}\right),
$$

where the correcting factor $F$ will be defined in Eq.(6.12). The natural unit of length is almost the inverse of $k_{*}$ :

$$
\delta_{*} \equiv\left(\tau_{*} \nu_{*}\right)^{1 / 2} .
$$

(The correcting factor in Eq.(1.8) is used in the bounds, but for a more intuitive understanding the reader should assume $D_{*}=\nu_{*}$.) In terms of these units, we can now explain our "sampling bound" of Theorem 6.7: Consider an $\varepsilon>0$, which will be the precision we want to achieve (up to a factor). Assume that two solutions of Eq.(1.1), $u$, and $v$ satisfy the bound

$$
\left|u\left(m \delta_{*}, t-n \tau_{*}\right)-v\left(m \delta_{*}, t-n \tau_{*}\right)\right| \leq \varepsilon,
$$


for all $n$ with $0 \leq n \leq C \log (1 / \varepsilon)$ and for all $|m| \leq(L+C / \varepsilon) / \delta_{*}$ where $C$ is some universal constant. There is another universal constant $C^{\prime}$ such that Eq.(1.10) implies

$$
|u(x, t)-v(x, t)| \leq C^{\prime} \varepsilon,
$$

for all $|x| \leq L$. Thus, a discrete sampling at spacings $\delta_{*}$ and $\tau_{*}$ suffices to bound the difference of the two functions everywhere in $\{|x| \leq L\}$. In other words, sampling in the inertial range for a time of order $\tau_{*} \log (1 / \varepsilon)$ suffices to bound the dissipative part of $u-v$ as a function of the sampling precision.

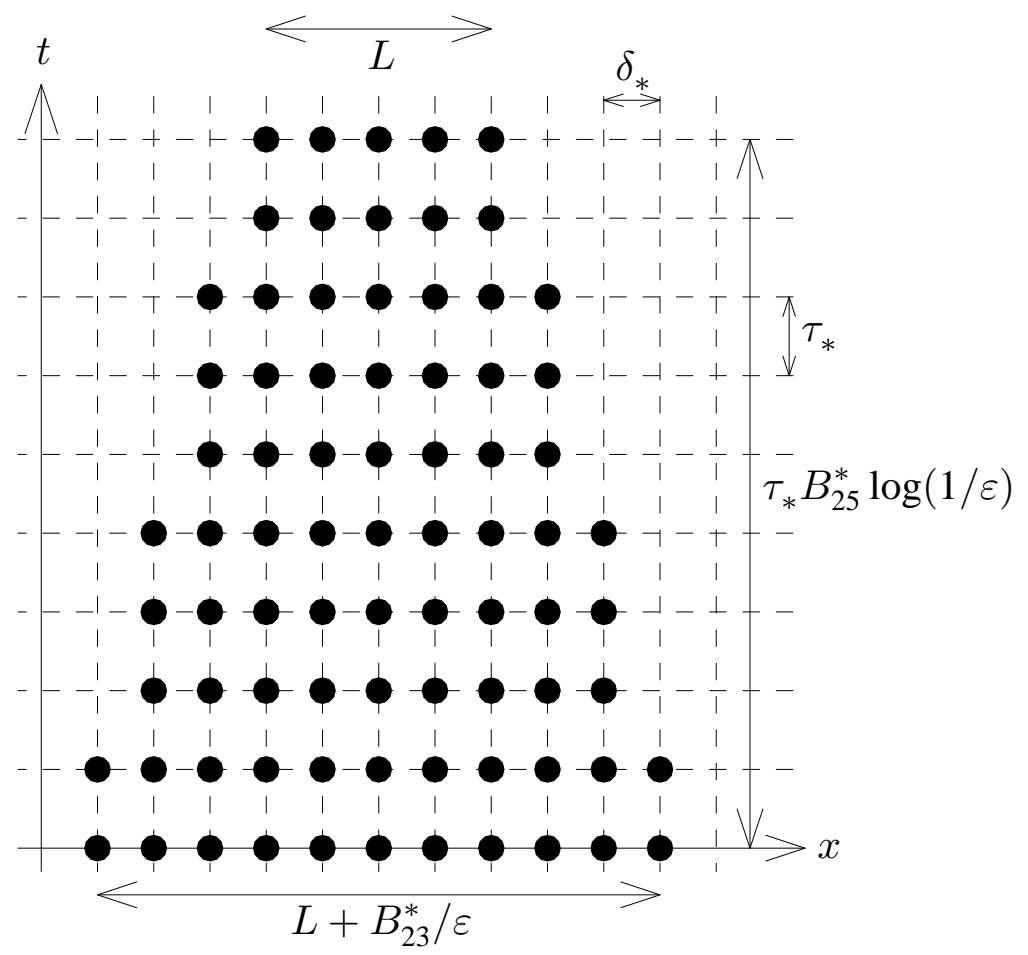

Fig. 1: A symbolic representation of the space-time points which need to be sampled with differences less than $\varepsilon$ to get a bound on the difference of two functions on the interval labeled $L$ to a precision $B_{25}^{*} \varepsilon$. See Theorem 6.7 for a definition of the constants $B_{23}^{*}, B_{24}^{*}$ and $B_{25}^{*}$.

This result is in line with our earlier bound [CE2] where we showed that, expressed in the units of the present paper, the number of balls of radius $\varepsilon$ in $\mathrm{L}^{\infty}$ needed to cover the global attractor (when restricted to $|x| \leq L$ ) has a bound of the order of

$$
\exp \left(C\left(L / \delta_{*}\right)^{d-1} \log (1 / \varepsilon)^{2}\right) \cdot \exp \left(C\left(L / \delta_{*}\right)^{d} \log (1 / \varepsilon)\right)
$$

One of the main ingredients of the proof of Eq.(1.11) is the "dissipative bound" of Theorem 5.1 below. In [CE2], we showed the inequality [CE2, Eq.(6.6)]:

$$
\sup _{|x| \leq \lambda-\varepsilon^{-1}}|w(x, t)| \leq C \varepsilon
$$


when $0 \leq t \leq \tau_{*}$, and $\sup _{|x| \leq \lambda}|w(x, 0)| \leq \varepsilon$. We can improve this bound (slightly) as follows and write it in natural units (see Eq.(5.8) for a precise formulation):

$$
\sup _{|x| \leq \lambda-\ell}\left|w\left(x, \tau_{*}\right)\right| \leq C\left(\sup _{|x| \leq \lambda}|w(x, 0)|+\left(\delta_{*} / \ell\right)\|w(\cdot, 0)\|_{\infty}\right)\left(\frac{D_{*}}{\nu_{*}}\right)^{(1+d) / 2} .
$$

Our improved bound in this paper exploits the dissipation as follows: Let $P_{>}$denote a localization of the Fourier transform of $w$ to wave numbers $k$ satisfying $|k|>k_{*}$ (see below for a more precise definition). Then,

$$
\sup _{|x| \leq \lambda-\ell}\left|P_{>} w\left(x, \tau_{*}\right)\right| \leq C\left(\frac{M_{*}}{\nu_{*} k_{*}^{2}}\right)^{1-d / 4}\left(\sup _{|x| \leq \lambda}|w(x, 0)|+\left(\delta_{*} / \ell\right)\|w(\cdot, 0)\|_{\infty}\right)\left(\frac{D_{*}}{\nu_{*}}\right)^{(1+d) / 2} .
$$

Thus, if $k_{*}$ is sufficiently large, the high frequency part of $w$ decays.

Our paper is organized as follows: We first show that the topological entropy per unit volume is defined and is bounded by the expansion rate. We then show the dissipative bound mentioned above, and finally show how it can be used to prove the sampling bound. From this, an estimate of the topological entropy per unit volume can be derived.

Acknowledgments. This work was partially supported by the Fonds National Suisse. Our collaboration was also made possible through the pleasant atmosphere at the IHES, Bures-surYvette.

\section{Existence of the Topological Entropy per Unit Volume}

We start by proving existence of the topological entropy per unit volume. This is somewhat similar to the standard proof of existence of topological entropy (see $[\mathrm{AKM}]$ ). We define the global attractor $\mathcal{A}$ by

$$
\mathcal{A}(B, \tau)=\bigcap_{n \geq 0} \Phi_{n \tau}(B) .
$$

Here, $B$ is a ball in $\mathrm{L}^{\infty}$ and $\Phi_{t}$ is the semi-flow defined by the evolution Eq.(1.1). It can be shown (see [MS]) that $\mathcal{A}(B, \tau)$ is invariant and that it does not depend on the initial ball $B$ (if it is large enough) nor on the (large enough) time $\tau \geq \tau_{0}(B)$. Thus, we define $\mathcal{A}=\mathcal{A}\left(B, \tau_{0}(B)\right)$.

For any $\varepsilon>0$ and any bounded set $Q$ in $\mathbf{R}^{d}$, whose boundary has Lebesgue measure 0 , we define $\mathcal{W}_{Q}^{\varepsilon}$ as the set of all finite coverings of $\mathcal{A}$ by open sets in $\mathrm{L}^{\infty}(Q)$ of diameter at most $\varepsilon$. Note that by the compactness of $\left.\mathcal{A}\right|_{Q}$, which follows from the uniform bounds on the gradient by Ascoli [MS], such finite coverings exist.

Let $\tau>0$ be a fixed time step, and let $T=n \tau$ with $n \in \mathbf{Z}$. For $\mathcal{U} \in \mathcal{W}_{Q}^{\varepsilon}$, we say that two trajectories $A_{1}$ and $A_{2}$ in $\mathcal{A}$ are $\mathcal{U}$-different before time $T$ if there is at least one $j, 0 \leq j \leq n$ for which the points $\Phi_{j \tau}\left(A_{1}\right)$ and $\Phi_{j \tau}\left(A_{2}\right)$ do not belong to the same element of $\mathcal{U}$. Let

$$
N_{T, \tau}(\mathcal{U})
$$


be the largest number of trajectories which are pairwise $\mathcal{U}$-different before time $T$ (and considered with time-step $\tau$.) Note that this number is finite since it is at most $(\operatorname{Card} \mathcal{U})^{T / \tau}$. Let

$$
N_{Q, T, \tau, \varepsilon}=\inf _{\mathcal{U} \in \mathcal{W}_{Q}^{\varepsilon}} N_{T, \tau}(\mathcal{U})
$$

Lemma 2.1. Let $Q_{1}$ and $Q_{2}$ be two bounded domains with boundary of zero Lebesgue measure and $Q_{1} \cap Q_{2}$ of zero Lebesgue measure. The functions $N_{Q, T, \tau, \varepsilon}$ satisfy the following bounds:

i) $N_{Q, T, \tau, \varepsilon}$ is non-increasing in $\varepsilon$.

ii) $N_{Q, T_{1}+T_{2}, \tau, \varepsilon} \leq N_{Q, T_{1}, \tau, \varepsilon} N_{Q, T_{2}, \tau, \varepsilon}$.

iii) $N_{Q_{1} \cup Q_{2}, T, \tau, \varepsilon} \leq N_{Q_{1}, T, \tau, \varepsilon} N_{Q_{2}, T, \tau, \varepsilon}$.

Proof. i) follows since $\mathcal{W}_{Q}^{\varepsilon}$ is non-decreasing in $\varepsilon$. For a given $\mathcal{U} \in \mathcal{W}_{Q}^{\varepsilon}$, we have easily from the definition

$$
N_{T_{1}+T_{2}, \tau}(\mathcal{U}) \leq N_{T_{1}, \tau}(\mathcal{U}) N_{T_{2}, \tau}(\mathcal{U})
$$

Indeed, if we consider a maximal collection of trajectories $A_{1}, A_{2}, \cdots, A_{N_{T_{1}+T_{2}, \tau}(\mathcal{U})}$, we can collect with $A_{1}$ all the remaining $A_{j}(j>1)$ whose step $\tau$ orbit $\mathcal{U}$ coincides with that of $A_{1}$ up to $T_{1}$. We know that since these orbits should differ between $T_{1}$ and $T_{2}$ their number is at most $N_{T_{2}, \tau}(\mathcal{U})$. We continue with the remaining orbits and this leads to at most $N_{T_{1}, \tau}(\mathcal{U})$ groups. ii) now follows immediately.

In order to prove iii), we consider $\mathcal{U}_{1} \in \mathcal{W}_{Q_{1}}^{\varepsilon}$ and $\mathcal{U}_{2} \in \mathcal{W}_{Q_{2}}^{\varepsilon}$. Since we are using the $\mathrm{L}^{\infty}$ norm, an argument similar to the one above leads to

$$
N_{T, \tau}\left(\mathcal{U}_{1} \times \mathcal{U}_{2}\right) \leq N_{T, \tau}\left(\mathcal{U}_{1}\right) N_{T, \tau}\left(\mathcal{U}_{2}\right)
$$

We also have easily (again because we are using the $\mathrm{L}^{\infty}$ norm)

$$
\mathcal{W}_{Q_{1}}^{\varepsilon} \times \mathcal{W}_{Q_{2}}^{\varepsilon} \subset \mathcal{W}_{Q_{1} \cup Q_{2}}^{\varepsilon}
$$

The claim iii) now follows easily.

Definition. Henceforth, we shall work with domains which are cubes, and we denote $Q_{L}$ the cube of side $L$ centered at the origin.

Theorem 2.2. The following limit exists

$$
h=\lim _{\varepsilon \rightarrow 0} \lim _{L \rightarrow \infty} \frac{1}{L^{d}} \lim _{T \rightarrow \infty} \frac{1}{T} \log N_{Q_{L}, T, \tau, \varepsilon} .
$$

Moreover, $h$ does not depend on $\tau$. It is called the topological entropy per unit volume of the system.

Proof. From ii) of the Lemma 2.1, and the usual subadditivity argument, we conclude that the following limit exists

$$
h_{Q, \tau, \varepsilon}^{(1)}=\lim _{T \rightarrow \infty} \frac{1}{T} \log N_{Q, T, \tau, \varepsilon} .
$$


Moreover, from i) it is non-increasing in $\varepsilon$ and from iii) it satisfies

$$
h_{Q_{1} \cup Q_{2}, \tau, \varepsilon}^{(1)} \leq h_{Q_{1}, \tau, \varepsilon}^{(1)}+h_{Q_{2}, \tau, \varepsilon}^{(1)} .
$$

Therefore the following limit exists

$$
h_{\tau, \varepsilon}^{(2)}=\lim _{L \rightarrow \infty} \frac{1}{L^{d}} h_{Q_{L}, \tau, \varepsilon}^{(1)} .
$$

Moreover, $h_{\tau, \varepsilon}^{(2)}$ is non-increasing in $\varepsilon$. This proves that the limit Eq.(2.2) exists.

We now show that it is independent of $\tau$. As in the treatment of topological entropy for finite dimensional systems, we start by giving an equivalent definition. Given a positive number $\zeta$, we say that two trajectories $A_{1}$ and $A_{2}$ in $\mathcal{A}$ are $\zeta$-separated in $Q$ before time $T$ (with time-steps $\tau$ ) if there exists an integer $j \in[0, T / \tau]$ for which

$$
\left\|\Phi_{j \tau}\left(A_{1}\right)-\Phi_{j \tau}\left(A_{2}\right)\right\|_{\mathrm{L}^{\infty}(Q)} \geq \zeta .
$$

We denote by $R_{Q, T, \tau}(\zeta)$ the maximum of the cardinalities of the sets of trajectories which are pairwise $\zeta$-different before time $T$. Since the coverings in $\mathcal{W}_{Q}^{\zeta}$ are of diameter less than $\zeta$, one has

$$
R_{Q, T, \tau}(\zeta) \leq N_{Q, T, \tau, \zeta / 2}
$$

Let now $\mathcal{U}$ be a finite covering of $\mathcal{A}$ by balls of radius $\zeta$ in $\mathrm{L}^{\infty}(Q)$. Then, if two trajectories differ on $\mathcal{U}$ at some time, their distance is at least $\zeta$. Therefore

$$
N_{Q, T, \tau, 2 \zeta} \leq R_{Q, T, \tau}(\zeta)
$$

These two estimates imply immediately that

$$
\lim _{\varepsilon \rightarrow 0} h_{\tau, \varepsilon}^{(2)}=\lim _{\varepsilon \rightarrow 0} \lim _{L \rightarrow \infty} \frac{1}{L^{d}} \lim _{T \rightarrow \infty} \frac{\log R_{Q_{L}, T, \tau}(\varepsilon)}{T} .
$$

Lemma 2.3. There are numbers $\gamma>0, \Gamma>1$ and $C<\infty$ such that for all $L, \varepsilon>0$ satisfying $L>C \varepsilon^{-1}$ and for all $A_{1}$ and $A_{2}$ in $\mathcal{A}$ for which $\left\|A_{1}-A_{2}\right\|_{L^{\infty}\left(Q_{L}\right)} \leq \varepsilon$, we have for any $0 \leq t \leq C^{-1} \varepsilon L-1$,

$$
\left\|\Phi_{t}\left(A_{1}\right)-\Phi_{t}\left(A_{2}\right)\right\|_{L^{\infty}\left(Q_{L-C \varepsilon^{-1}(t+1)}\right)} \leq \Gamma e^{\gamma t} \varepsilon
$$

with $\Gamma>1$.

Proof. See Theorem 5.1, Eq.(5.8) below. In fact, the statement was already shown in [CE2]. Using the above estimate one has easily for $\tau^{\prime}<\tau$,

$$
R_{Q_{L}, T, \tau^{\prime}}(\varepsilon) \leq R_{Q_{L+C \varepsilon^{-1}(\tau+1)}, T, \tau}\left(\varepsilon \Gamma^{-1} e^{-\gamma \tau}\right)
$$

and

$$
R_{Q_{L}, T, \tau}(\varepsilon) \leq R_{Q_{L+C \varepsilon^{-1}(\tau+1)}, T, \tau^{\prime}}\left(\varepsilon \Gamma^{-1} e^{-\gamma \tau}\right) .
$$

It follows now immediately from Eq.(2.3) that $\lim _{\varepsilon \rightarrow 0} h_{\varepsilon, \tau}^{(2)}$ does not depend on $\tau$. This concludes the proof of Theorem 2.2. 


\section{Upper Bound on the Entropy per Unit Volume}

It does not follow from what was said in the previous section that we have defined a finite number. We now give an upper bound. We first observe that from the sub-additivity argument we have

$$
\lim _{T \rightarrow \infty} \frac{1}{T} \log R_{Q_{L}, T, \tau}(\varepsilon)=\inf _{T} \frac{1}{T} \log N_{Q_{L}, T, \tau, \varepsilon} .
$$

Therefore, in order to obtain an upper bound, we can fix a $T$ and vary $L$.

Let $H_{\varepsilon}$ denote the $\varepsilon$-entropy per unit volume defined in [KT], (see also [CE2] for the application to the case at hand). This means that we first define $N_{Q}(\varepsilon)$ as the minimum number of balls of radius $\varepsilon$ in $\mathrm{L}^{\infty}(Q)$ needed to cover $\left.\mathcal{A}\right|_{Q}$ (the functions on the attractor $\mathcal{A}$ restricted to $Q$ ), and then

$$
H_{\varepsilon}=\lim _{L \rightarrow \infty} \frac{\log N_{Q_{L}}(\varepsilon)}{L^{d}} .
$$

We define the upper dimension per unit volume $d_{\text {up }}$ of $\mathcal{A}$ by

$$
d_{\text {up }}=\limsup _{\varepsilon \rightarrow 0} \frac{H_{\varepsilon}}{\log (1 / \varepsilon)} \text {. }
$$

Note that it follows from [CE2] that $d_{\text {up }}$ is finite. It is an open question to prove that the limit exists. However, we have a bound:

Theorem 3.1. The topological entropy per unit volume is bounded by

$$
h \leq d_{\text {up }} \gamma
$$

where $\gamma$ is the constant appearing in Lemma 2.3.

Remark. In terms of the variables which scale like the system, we have $\gamma=M_{*}$.

Proof. Let $\eta>0$ be a fixed number. Let $\varepsilon_{0}>0$ be small enough such that for all $\varepsilon \in\left(0, \varepsilon_{0}\right)$ we have

$$
\frac{H_{\varepsilon}}{\log (1 / \varepsilon)} \leq d_{\text {up }}+\eta \text {. }
$$

For a fixed $\varepsilon \in\left(0, \varepsilon_{0}\right)$ let $L_{\eta, \varepsilon}>0$ be such that for any $L>L_{\eta, \varepsilon}$ we have,

$$
\left|\frac{\log N_{Q_{L}}(\varepsilon)}{L^{d}}-H_{\varepsilon}\right| \leq \eta \text {. }
$$

We now give an upper bound on $R_{Q_{L}, T, \tau}(\zeta)$. For $\varepsilon \in\left(0, \varepsilon_{0}\right)$, choose a finite covering $\mathcal{U}$ of $\mathcal{A}$ in $\mathrm{L}^{\infty}\left(Q_{L+(T+1) \varepsilon^{-1}}\right)$ by balls of radius $\varepsilon \Gamma^{-1} e^{-\gamma T} / 2$. By [CE2] and the previous discussion, we know that we can find such a covering $\mathcal{U}$ of cardinality at most

$$
\exp \left(\left(L+C \varepsilon^{-1}(T+1)\right)^{d} \cdot\left(\eta+\left(d_{\text {up }}+\eta\right)\left(\gamma T+\log \left(2 \Gamma \varepsilon^{-1}\right)\right)\right)\right)
$$


Moreover, from the definition of $\gamma$, if $A_{1}$ and $A_{2}$ belong to the same element of $\mathcal{U}$, it follows from Lemma 2.3 that

$$
\sup _{0 \leq t \leq T}\left\|\Phi_{t}\left(A_{1}\right)-\Phi_{t}\left(A_{2}\right)\right\|_{\mathrm{L}^{\infty}\left(Q_{L}\right)} \leq \varepsilon
$$

Therefore,

$$
R_{Q, T, \tau}(\varepsilon) \leq \exp \left(\left(L+C \varepsilon^{-1}(T+1)\right)^{d} \cdot\left(\eta+\left(d_{\text {up }}+\eta\right)\left(\gamma T+\log \left(2 \Gamma \varepsilon^{-1}\right)\right)\right)\right)
$$

It follows that

$$
\begin{gathered}
\lim _{L \rightarrow \infty} \frac{1}{L^{d}} \inf _{T} \frac{1}{T} \log R_{Q_{L}, T, \tau}(\varepsilon) \leq \lim _{L \rightarrow \infty} \frac{1}{L^{d}} \frac{1}{T} \log R_{Q_{L}, T, \tau}(\varepsilon) \\
\leq \eta+\left(d_{\text {up }}+\eta\right)\left(\gamma+T^{-1} \log \left(2 \Gamma \varepsilon^{-1}\right)\right)
\end{gathered}
$$

and the result follows by letting $T \rightarrow \infty$ and then $\eta \rightarrow 0$.

\section{Localization in Momentum Space and Bounds on the Semi- Group}

In this section, we deal with some simple bounds on the semi-group generated by $D \Delta$. We begin by constructing the localization in momentum space. Let $\chi \geq 0$ be a smooth function with support in $|k| \leq 2$ and which is equal to 1 for $|k| \leq 1$. We also assume $\chi \leq 1$. We shall denote $\chi_{k<k_{*}}=\chi\left(k / k_{*}\right)$ and $\chi_{k>k_{*}}=1-\chi\left(k / k_{*}\right)$. We define the convolution operators $\mathcal{G}_{\tau}$, $\mathcal{G}_{\tau,>}$ and $\mathcal{G}_{\tau,<}^{*}$ by

$$
\begin{aligned}
\mathcal{G}_{\tau}(x) & =\int \mathrm{d} k e^{i k x-D k^{2} \tau}, \\
\mathcal{G}_{\tau,>}(x) & =\int \mathrm{d} k e^{i k x} e^{-D k^{2} \tau} \chi_{k>k_{*}}, \\
\mathcal{G}_{\tau,<}(x) & =\int \mathrm{d} k e^{i k x} e^{-D k^{2} \tau} \chi_{k<k_{*}}=\mathcal{G}_{\tau}(x)-\mathcal{G}_{\tau,>}(x) .
\end{aligned}
$$

Notation. The constants $B_{0}^{*}, B_{1}^{*}, \ldots$, do only depend on the quotient $D_{*} / \nu_{*}$ (something like the condition number of the matrix $D$ ), but not on any other parameters of the problem, except $d$. We also recall that the constants $C_{0}, \ldots$, are numerical factors which do not depend on any parameters of the problem except $d$.

We now state and prove various estimates on these kernels.

Lemma 4.1. For every $p \geq 0$ there is a constant $B_{0}^{*}(p)$ such that for all $\tau>0$ one has the bound

$$
\left\|\int \mathrm{d} k e^{i k x} e^{-D k^{2} \tau}\right\| \leq B_{0}^{*}(p) \frac{1}{\left(\nu_{*} \tau\right)^{d / 2}\left(1+\frac{|x|^{2}}{D_{*} \tau}\right)^{p / 2}} .
$$


Furthermore, convolution with $\mathcal{G}_{\tau}$ is a well-defined operator on bounded functions and has norm (as a map from $\mathrm{L}^{\infty}$ to itself) bounded by

$$
\left\|\mathcal{G}_{\tau}\right\|_{\infty} \leq B_{1}^{*}
$$

for some $B_{1}^{*}$.

Proof. For simplicity, we write the proof for the case of $d=1$, but with a distinction of the upper and lower bounds $\left(D_{*}\right.$, resp. $\left.\nu_{*}\right)$ on the matrix $D$. For example

$$
\begin{aligned}
x \int \mathrm{d} k e^{i k x} e^{-D k^{2} \tau} & =\int \mathrm{d} k\left(-i \partial_{k} e^{i k x}\right) e^{-D k^{2} \tau} \\
& =-i \int \mathrm{d} k e^{i k x} e^{-D k^{2} \tau} 2 D k \tau
\end{aligned}
$$

Thus, in this case,

$$
\begin{aligned}
\left|\int \mathrm{d} k e^{i k x} e^{-D k^{2} \tau}\right| & \leq|x|^{-1} \int \mathrm{d} k e^{-D k^{2} \tau} 2 D|k| \tau \\
& \leq C_{0}\left(\frac{D_{*} \tau}{|x|^{2}}\right)^{1 / 2} \frac{1}{\left(\nu_{*} \tau\right)^{1 / 2}}\left(\frac{D_{*}}{\nu_{*}}\right)^{1 / 2} .
\end{aligned}
$$

Using $D_{*} / \nu_{*} \geq 1$, the generalization to arbitrary $d$ and $p$ is easy and is left to the reader. The second assertion follows by taking $p=d+2$ and integrating. The reader can also check that $B_{1}^{*}=C_{1}\left(D_{*} / \nu_{*}\right)^{d+2}$, for some $C_{1}$.

Lemma 4.2. Let $\tau \geq 0$. For all $p \geq 0$ there is a constant $B_{2}^{*}(p)$ such that for all $z \in \mathbf{C}^{d}$ one has a bound

$$
\left\|\int \mathrm{d} k e^{i k z} e^{-D k^{2} \tau} \chi_{k<k_{*}}\right\| \leq B_{2}^{*}(p) \frac{1}{\left(k_{*}^{-2}+\nu_{*} \tau\right)^{d / 2}} \frac{e^{2 k_{*}|\operatorname{Im} z|}}{\left(1+\frac{|z|^{2}}{k_{*}^{-2}+D_{*} \tau}\right)^{p / 2}},
$$

where $|\operatorname{Im} z| \equiv \sum_{i=1}^{d}\left|\operatorname{Im} z_{i}\right|$.

Proof. We get, for $z \in \mathbf{C}$,

$$
z^{p} \int \mathrm{d} k e^{i k z} e^{-D k^{2} \tau} \chi_{k<k_{*}}=\int \mathrm{d} k\left(\left(-i \partial_{k}\right)^{p} e^{i k z}\right) e^{-D k^{2} \tau} \chi\left(k / k_{*}\right),
$$

and integrating by parts this leads to a finite sum of terms of the form

$$
\mathcal{O}(1) \int \mathrm{d} k e^{i k z} e^{-D k^{2} \tau}(D \tau k)^{n_{1}} k^{-n_{2}} k_{*}^{-n_{3}}\left(\partial_{k}^{n_{3}} \chi\right)\left(k / k_{*}\right)
$$


where $n_{1}+n_{2}+n_{3}=p$, with $n_{2} \leq n_{1}$. We let $f=\partial_{k}^{n_{3}} \chi$, and we write $n_{1}=s_{1}+s_{2}, n_{2}=s_{1}$, and $n_{3}=s_{3}$, where now $s_{i} \geq 0$ and $p=2 s_{1}+s_{2}+s_{3}$. Thus we need to bound expressions of the form

$$
\int_{|k| \leq 2 k_{*}} \mathrm{~d} k e^{-D k^{2} \tau}(D \tau)^{s_{1}+s_{2}} k^{s_{2}} k_{*}^{-s_{3}} f(k) .
$$

It will be useful to consider first the case $\nu_{*} \tau<1 / k_{*}^{2}$. Then we can bound (4.5) in $d$ dimensions by

$$
\mathcal{O}(1)\left(\frac{D_{*}}{\nu_{*}}\right)^{p} k_{*}^{d}\left(\nu_{*} \tau\right)^{s_{1}+s_{2}} k_{*}^{s_{2}} k_{*}^{-s_{3}} \leq \mathcal{O}(1)\left(\frac{D_{*}}{\nu_{*}}\right)^{p} k_{*}^{d} k_{*}^{-2 s_{1}-2 s_{2}} k_{*}^{s_{2}} k_{*}^{-s_{3}}=\mathcal{O}(1)\left(\frac{D_{*}}{\nu_{*}}\right)^{p} k_{*}^{d-p} .
$$

In the case when $\nu_{*} \tau \geq 1 / k_{*}^{2}$, we bound (4.5) by

$$
\begin{aligned}
\mathcal{O}(1)\left(\frac{D_{*}}{\nu_{*}}\right)^{p} \int \mathrm{d} k & e^{-\nu_{*} k^{2} \tau}\left(\nu_{*} \tau\right)^{s_{1}+s_{2}}|k|^{s_{2}}\left(\nu_{*} \tau\right)^{s_{3} / 2} \\
\leq & \mathcal{O}(1)\left(\frac{D_{*}}{\nu_{*}}\right)^{p}\left(\nu_{*} \tau\right)^{-d / 2+s_{1}+s_{2}-s_{2} / 2+s_{3} / 2} \\
& =\mathcal{O}(1)\left(\frac{D_{*}}{\nu_{*}}\right)^{p}\left(\nu_{*} \tau\right)^{-d / 2+p / 2} .
\end{aligned}
$$

Combining (4.6) and (4.7), and observing that $\left|e^{i k z}\right| \leq e^{2 k_{*}|\operatorname{Im} z|}$ on the support of $\chi_{k<k_{*}}$, we conclude the proof of Lemma 4.2.

Lemma 4.3. For every $p \geq 0$ there is a constant $B_{3}^{*}(p)$ such that for all $k_{*}>0$ and all $\tau>0$, one has the following bounds:

i) When $\nu_{*} \tau>1 / k_{*}^{2}$ one has

$$
\left\|\int \mathrm{d} k e^{i k x} e^{-D k^{2} \tau} \chi_{k>k_{*}}\right\| \leq B_{3}^{*}(p) \frac{e^{-\nu_{*} k_{*}^{2} \tau / 2}}{\left(\nu_{*} \tau\right)^{d / 2}\left(1+\frac{|x|^{2}}{D_{*} \tau}\right)^{p / 2}} .
$$

ii) When $\nu_{*} \tau \leq 1 / k_{*}^{2}$ one has

$$
\begin{aligned}
\left\|\int \mathrm{d} k e^{i k x} e^{-D k^{2} \tau} \chi_{k>k_{*}}\right\| & \leq B_{3}^{*}(p) e^{-\nu_{*} k_{*}^{2} \tau / 2} \\
& \cdot\left(\frac{1}{\left(\nu_{*} \tau\right)^{d / 2}\left(1+\frac{|x|^{2}}{D_{*} \tau}\right)^{p / 2}}+\frac{1}{k_{*}^{-d}\left(1+k_{*}^{2}|x|^{2}\right)^{p / 2}}\right) .
\end{aligned}
$$

Proof. Assume first that $\nu_{*} \tau \leq 1 / k_{*}^{2}$. In that case, $e^{\nu_{*} k_{*}^{2} \tau} \leq e$, and thus it suffices to produce a bound without exponential factor. We can write $\chi_{k>k_{*}}=1-\chi_{k<k_{*}}$ and get a bound by 
combining Eq.(4.1) with Eq.(4.4). This leads to

$$
\begin{aligned}
\left\|\int \mathrm{d} k e^{i k z} e^{-D k^{2} \tau} \chi_{k>k_{*}}\right\| & \leq e B_{0}^{*}(p) \frac{1}{\left(\nu_{*} \tau\right)^{d / 2}\left(1+\frac{|x|^{2}}{D_{*} \tau}\right)^{d / 2}}\left(\frac{D_{*}}{\nu_{*}}\right)^{p / 2} \\
& +e B_{2}^{*}(p) \frac{1}{\left(k_{*}^{-2}+\nu_{*} \tau\right)^{d / 2}} \frac{1}{\left(1+\frac{|x|^{2}}{k_{*}^{-2}+D_{*} \tau}\right)^{p / 2}}\left(\frac{D_{*}}{\nu_{*}}\right),
\end{aligned}
$$

from which the first assertion follows. In the case $\nu_{*} \tau \geq 1 / k_{*}^{2}$, we integrate again by parts and get to bound an expression of the form (we work again in the case $d=1$ only):

$$
\begin{aligned}
x \int \mathrm{d} k e^{i k x} e^{-D k^{2} \tau} \chi_{k>k_{*}} & \\
& =-i \int \mathrm{d} k e^{i k x} e^{-D k^{2} \tau}\left(2 D k\left(1-\chi\left(k / k_{*}\right)\right)-k_{*}^{-1}\left(\partial_{k} \chi\right)\left(k / k_{*}\right)\right) .
\end{aligned}
$$

Therefore, we get, as in (4.3),

$$
\begin{aligned}
\left|\int \mathrm{d} k e^{i k x} e^{-D k^{2} \tau} \chi_{k>k_{*}}\right| & \leq|x|^{-1} \mathcal{O}(1) e^{-\nu_{*} k^{2} \tau / 2} \int \mathrm{d} k e^{-D k^{2} \tau / 2}|2 D k \tau| \\
& +|x|^{-1} \mathcal{O}(1) e^{-\nu_{*} k^{2} \tau / 2} \int \mathrm{d} k e^{-D k^{2} \tau / 2}\left|k_{*}^{-1} \chi^{\prime}\left(k / k_{*}\right)\right| \\
& \leq C e^{-\nu_{*} k^{2} \tau / 2}\left(\frac{D_{*} \tau}{|x|^{2}}\right)^{1 / 2} \frac{1}{\left(\nu_{*} \tau\right)^{1 / 2}}\left(\frac{D_{*}}{\nu_{*}}\right)^{1 / 2} \\
& +C e^{-\nu_{*} k^{2} \tau / 2} \frac{1}{\left(\nu_{*} \tau\right)^{1 / 2}} \frac{k_{*}^{-1}}{|x|} \\
& \leq C e^{-\nu_{*} k^{2} \tau / 2}\left(\frac{D_{*} \tau}{|x|^{2}}\right)^{1 / 2} \frac{1}{\left(\nu_{*} \tau\right)^{1 / 2}}\left(\frac{D_{*}}{\nu_{*}}\right)^{1 / 2} .
\end{aligned}
$$

The generalization to arbitrary $p$ and $d$ is easy and is left to the reader.

\section{The Dissipative Bound}

In this section, we consider in detail the equation ${ }^{1}$

$$
\dot{w}(x, t)=D \Delta w(x, t)+M(x, t) w(x, t),
$$

where $w$ takes values in $\mathbf{R}^{N}$. Our bounds will work in dimensions $d \leq 3$. We first state the assumptions of the Introduction in a more precise form. We first assume that

$$
\|M(x, t)\| \leq M_{*},
$$

\footnotetext{
${ }^{1}$ For simplicity we assume isotropy of the diffusion in the $d$ components of the coordinates, but this requirement could be dropped if desired.
} 
for all $x \in \mathbf{R}^{d}, t \in \mathbf{R}_{+}$. We assume further that

$$
|w(x, t)| \leq Q_{*},
$$

for all $x, t$. (This is the reason for the choice of $Q_{*} / 2$ in the bound on $u$.) Here, and in the remainder of the paper, $|\cdot|$ is the $l^{2}$-norm of a vector in $\mathbf{R}^{N}$.

We will fix the constant $k_{*}$ only in the next section. But we will work here with the following "comparisons of scales" which will be essential in the bounds:

$$
\begin{aligned}
& \tau_{*}=1 / M_{*}, \\
& \delta_{*}^{2}=D_{*} \tau_{*}, \\
& k_{*}^{2} \geq 1 /\left(\nu_{*} \tau_{*}\right) .
\end{aligned}
$$

We consider next the integral representation of $w_{1}(\cdot) \equiv w\left(\cdot, t=\tau_{*}\right)$ :

$$
w\left(x, \tau_{*}\right)=\left(\mathcal{G}_{\tau_{*}} \star w\right)(x)+\int_{0}^{\tau_{*}} \mathrm{~d} s\left(\mathcal{G}_{\tau_{*}-s} \star(M(\cdot, s) w(\cdot, s))\right)(x) \equiv w_{1}^{(1)}+w_{1}^{(2)} .
$$

Using the decomposition $\mathcal{G}_{\tau}=\mathcal{G}_{\tau,<}+\mathcal{G}_{\tau,>}$, we split $w_{1}^{(1)}$ and $w_{1}^{(2)}$ into high and low frequency parts:

$$
w_{1,<}=w_{1,<}^{(1)}+w_{1,<}^{(2)}, \quad w_{1,>}=w_{1,>}^{(1)}+w_{1,>}^{(2)} .
$$

Note that $w_{<}$has Fourier components in $\left\{|k| \leq 2 k_{*}\right\}$ and $w_{>}$has components in $\left\{|k| \geq k_{*}\right\}$.

Theorem 5.1. (Dissipative bound) In dimension $d \leq 3$, we have the following bounds for some constants $B_{4}^{*}$ and $B_{5}^{*}$ : Upon localizing in space, we have for $0 \leq \tau \leq \tau_{*}$,

$$
\sup _{|x| \leq \lambda-\ell}|w(x, \tau)| \leq B_{4}^{*}\left(\sup _{|x| \leq \lambda}|w(x, 0)|+\left(\delta_{*} / \ell\right)\|w(\cdot, 0)\|_{\infty}\right) .
$$

Localizing in position space and momentum space, we have

$$
\begin{aligned}
& \sup _{|x| \leq \lambda-\ell}\left|w_{>}\left(x, \tau_{*}\right)\right| \\
& \leq B_{5}^{*}\left(e^{-\left(\nu_{*} k_{*}^{2} / M_{*}\right) / 2}+\left(\frac{M_{*}}{\nu_{*} k_{*}^{2}}\right)^{1-d / 4}\right) \\
& \cdot\left(\sup _{|x| \leq \lambda}|w(x, 0)|+\left(\delta_{*} / \ell\right)\|w(\cdot, 0)\|_{\infty}\right) .
\end{aligned}
$$

Proof. We define, as in [CE1], a family of space cutoff functions: Let

$$
\psi_{a}(x)=\frac{Z}{1+\left((x-a)^{2} / \delta_{*}^{2}\right)^{1+d / 2}}=\psi(x-a),
$$


where $Z=\delta_{*}^{-d} / \int \mathrm{d} x\left(1+x^{2}\right)^{-1-d / 2}$ is chosen such that $\int \mathrm{d} x \psi(x)=1$. We start with a bound in $\mathrm{L}^{2}$ :

Lemma 5.2. There is a constant $B_{6}^{*}$ such that the solution of Eq.(5.1) satisfies:

$$
\sup _{0 \leq t \leq \tau_{*}} \sup _{|a| \leq \lambda-\ell} \int \mathrm{d} x \psi_{a}(x)|w(x, t)|^{2} \leq B_{6}^{*}\left(\sup _{|x|<\lambda}|w(x, 0)|^{2}+\|w(\cdot, 0)\|_{\infty}^{2}\left(\delta_{*} / \ell\right)^{2}\right) .
$$

Proof. Let

$$
X_{t}=\int \mathrm{d} x \psi_{a}(x)|w(x, t)|^{2} .
$$

Then we have, from the equations of motion,

$$
\begin{aligned}
\partial_{t} X_{t} & =\int \mathrm{d} x \psi_{a}(w \cdot(D \Delta w+M w)+(D \Delta w+M w) \cdot w) \\
& =-2 \int \mathrm{d} x \psi_{a}(\nabla w) \cdot D_{\text {sym }} \nabla w+2 \int \mathrm{d} x \psi_{a} M_{\text {sym }}|w|^{2}-\int \mathrm{d} x\left(\nabla \psi_{a}\right) \nabla w \cdot D w
\end{aligned}
$$

Here, $M_{\text {sym }}=\left(M+M^{\mathrm{T}}\right) / 2$ and $\left\|M_{\text {sym }}\right\| \leq\|M\| \leq M_{*}$. Observe that by our choice of $\psi$ we have $|\nabla \psi| \leq C_{2} \psi / \delta_{*}$ for some constant $C_{2}$ independent of $\delta_{*}$. Using the definitions (1.3) of $\nu_{*}$ and $D_{*}$, we find

$$
\left|\partial_{t} X_{t}\right| \leq-2 \nu_{*} \int \mathrm{d} x \psi_{a}|\nabla w|^{2}+2 \int \mathrm{d} x \psi_{a} M_{*}|w|^{2}+2 C_{2} \int \mathrm{d} x\left(D_{*} / \delta_{*}\right) \psi_{a}|w \cdot \nabla w| .
$$

We polarize the term containing $w \cdot \nabla w$ and use the identity $\left(D_{*} / \delta_{*}\right)^{2} / \nu_{*}=M_{*}$. see Eq.(5.5), Then we see that we can find a constant $C_{3}$ such that

$$
\left|\partial_{t} X_{t}\right| \leq C_{3} M_{*}\left(1+\left(D_{*} / \nu_{*}\right)\right) X_{t}
$$

Coming back to the assumptions of Lemma 5.2, we see that when $|a| \leq \lambda-\ell$ we have

$$
\begin{aligned}
X_{0}=\int \mathrm{d} x \psi_{a}\left|w_{0}\right|^{2} & =\int_{|x| \leq \lambda} \mathrm{d} x \psi_{a}\left|w_{0}\right|^{2}+\int_{|x| \geq \lambda} \mathrm{d} x \psi_{a}\left|w_{0}\right|^{2} \\
& \leq \sup _{|x|<\lambda}|w(x, 0)|^{2}+C_{4}\left\|w_{0}\right\|_{\infty}^{2}\left(\frac{\delta_{*}}{\ell}\right)^{2} .
\end{aligned}
$$

Using this bound on the initial condition, the differential inequality (5.11), and $\tau_{*} M_{*}=1$, the assertion of Lemma 5.2 follows with $B_{6}^{*}=\exp \left(C_{3}\left(1+\left(D_{*} / \nu_{*}\right)\right)\right)$.

We begin the proof of (5.9). We deal first with the bound on $w_{1,>}^{(2)}(x)$, when $|x| \leq \lambda-\ell$. Consider, for $\tau_{*} \geq t \geq s \geq 0$, the quantity

$$
\begin{aligned}
Y_{t, s} & =\int \mathrm{d} y \mathcal{G}_{t-s,>}(x-y) M(y, s) w(y, s) \\
& =\int \mathrm{d} y \frac{\mathcal{G}_{t-s,>}(x-y)}{\sqrt{\psi(x-y)}} \sqrt{\psi(x-y)} M(y, s) w(y, s) .
\end{aligned}
$$


We consider first the case $\nu_{*} \tau>k_{*}^{-2}$ : Then, by the Schwarz inequality and Eq.(4.8), we have

$$
\begin{aligned}
Y_{t, s}^{2} & \leq \int \mathrm{d} y \frac{\left|\mathcal{G}_{t-s,>}(x-y)\right|^{2}}{\psi(x-y)} M_{*}^{2} \int \mathrm{d} z \psi_{x}(z)|w(z, s)|^{2} \\
& \leq \int \mathrm{d} y \frac{B_{3}^{*}(p)^{2} e^{-\nu_{*} k_{*}^{2}(t-s)}\left(\frac{D_{*}}{\nu_{*}}\right)^{2 p}}{\left(\nu_{*}(t-s)\right)^{d}\left(1+\frac{|x-y|^{2}}{D_{*}(t-s)}\right)} \frac{1+\left(|x-y|^{2} / \delta_{*}^{2}\right)^{1+d / 2}}{Z} \\
& \cdot M_{*}^{2} \int \mathrm{d} z \psi_{x}(z)|w(z, s)|^{2} .
\end{aligned}
$$

Since we deal with $|x| \leq \lambda-\ell$, we get from (5.10),

$$
\begin{aligned}
Y_{t, s}^{2} & \leq B_{3}^{*}(p)^{2}\left(\frac{D_{*}}{\nu_{*}}\right)^{2 p} Z^{-1} \int \mathrm{d} y \frac{e^{-\nu_{*} k_{*}^{2}(t-s)}}{\nu_{*}^{d}(t-s)^{d}}\left(1+\frac{|x-y|^{2}}{D_{*}(t-s)}\right)^{-p}\left(1+\frac{|x-y|^{2}}{\delta_{*}^{2}}\right)^{1+d / 2} \\
& \cdot M_{*}^{2} B_{6}^{*}\left(K^{2}+Q_{*}^{2}\left(\frac{\delta_{*}}{\ell}\right)^{2}\right)
\end{aligned}
$$

where $K=\sup _{|x| \leq \lambda}|w(x, 0)|$ and $Q_{*} \geq\|w(\cdot, 0)\|_{\infty}$. Note now that by Eq.(5.5),

$$
\delta_{*}^{2}=D_{*} \tau_{*} \geq D_{*}(t-s),
$$

since $\tau_{*} \geq t \geq s \geq 0$. Therefore,

$$
\begin{aligned}
Y_{t, s}^{2} & \leq B_{3}^{*}(p)^{2}\left(\frac{D_{*}}{\nu_{*}}\right)^{2 p} Z^{-1} \int \mathrm{d} y \frac{e^{-\nu_{*} k_{*}^{2}(t-s)}}{\nu_{*}^{d}(t-s)^{d}}\left(1+\frac{|x-y|^{2}}{D_{*}(t-s)}\right)^{1+d / 2-p} \\
& \cdot M_{*}^{2} B_{6}^{*}\left(K^{2}+Q_{*}^{2}\left(\frac{\delta_{*}}{\ell}\right)^{2}\right) .
\end{aligned}
$$

Taking $p=d+2$, integrating over $y$, and using again $\delta_{*}^{2}=D_{*} / M_{*}$, we get, for some constant $B_{7}^{*}$ :

$$
\begin{aligned}
Y_{t, s}^{2} & \leq C_{5} B_{3}^{*}(p)^{2}\left(\frac{D_{*}}{\nu_{*}}\right)^{2 d+4} \delta_{*}^{d} D_{*}^{d / 2}(t-s)^{d / 2} \frac{e^{-\nu_{*} k_{*}^{2}(t-s)}}{\nu_{*}^{d}(t-s)^{d}} M_{*}^{2} B_{6}^{*}\left(K+Q_{*} \frac{\delta_{*}}{\ell}\right)^{2} \\
& =\left(B_{7}^{*}\right)^{2} M_{*}^{2-d / 2}\left(K+Q_{*} \frac{\delta_{*}}{\ell}\right)^{2} \frac{e^{-\nu_{*} k_{*}^{2}(t-s)}}{\nu_{*}^{d / 2}(t-s)^{d / 2}} .
\end{aligned}
$$

Taking the square root of this bound, integrating over $s$, (and using at this point the hypothesis $d \leq 3$ ) we get for all $\tau \in\left[0, \tau_{*}\right]$ and all $|x| \leq \lambda-\ell$,

$$
\begin{aligned}
\left|w_{>}^{(2)}(x, \tau)\right| & \leq \int_{0}^{\tau} \mathrm{d} s\left|Y_{\tau, s}\right| \leq \int_{0}^{\infty} \mathrm{d} s B_{7}^{*} M_{*}^{1-d / 4}\left(\nu_{*} k_{*}^{2}\right)^{d / 4}\left(K+Q_{*} \frac{\delta_{*}}{\ell}\right) \frac{e^{-\nu_{*} k_{*}^{2} s / 2}}{\left(\nu_{*} k_{*}^{2} s\right)^{d / 4}} \\
& \leq B_{8}^{*}\left(\frac{M_{*}}{\nu_{*} k_{*}^{2}}\right)^{1-d / 4}\left(K+Q_{*} \frac{\delta_{*}}{\ell}\right) .
\end{aligned}
$$


This completes the study of the contribution of $w_{1,>}^{(2)}$ to the bound of Eq.(5.9), when $\nu_{*} \tau>1 / k_{*}^{2}$.

We next deal with the case $\nu_{*} \tau \leq 1 / k_{*}^{2}$. The contribution corresponding to the first term of Eq.(4.9) is treated as before noting that in Eq.(5.13) we actually integrate over all $\tau$, and that the inequality $\nu_{*} \tau>1 / k_{*}^{2}$ was not used anywhere except for being able to use (4.8) which is the same as the first term in (4.9). Thus, we consider here only the second term. Although the bounds are quite similar to the previous case, it might be better to actually spell them out. By the Schwarz inequality and Eq.(4.9), we now bound

$$
\begin{aligned}
W_{t, s}^{2} & =\int \mathrm{d} y \frac{B_{3}^{*}(p)^{2} e^{-\nu_{*} k_{*}^{2}(t-s)}\left(\frac{D_{*}}{\nu_{*}}\right)^{2 p}}{k_{*}^{-2 d}\left(1+k_{*}^{2}|x-y|^{2}\right)^{p}} \frac{1+\left(|x-y|^{2} / \delta_{*}^{2}\right)^{1+d / 2}}{Z} \\
& \cdot M_{*}^{2} \int \mathrm{d} z \psi_{x}(z)|w(z, s)|^{2} .
\end{aligned}
$$

Since we still deal with $|x| \leq \lambda-\ell$, we get from (5.10),

$$
\begin{aligned}
W_{t, s}^{2} & \leq B_{3}^{*}(p)^{2}\left(\frac{D_{*}}{\nu_{*}}\right)^{2 p} Z^{-1} \int \mathrm{d} y \frac{e^{-\nu_{*} k_{*}^{2}(t-s)}}{k_{*}^{-2 d}}\left(1+k_{*}^{2}|x-y|^{2}\right)^{-p}\left(1+\frac{|x-y|^{2}}{\delta_{*}^{2}}\right)^{1+d / 2} \\
& \cdot M_{*}^{2} B_{6}^{*}\left(K^{2}+Q_{*}^{2}\left(\frac{\delta_{*}}{\ell}\right)^{2}\right) .
\end{aligned}
$$

Note now that by Eq.(5.5),

$$
\delta_{*}^{2}=D_{*} / M \geq k_{*}^{-2}
$$

This leads to

$$
\begin{aligned}
W_{t, s}^{2} & \leq\left(B_{3}^{*}(p)\right)^{2}\left(\frac{D_{*}}{\nu_{*}}\right)^{2 p} Z^{-1} \int \mathrm{d} y k_{*}^{2 d}\left(1+k_{*}^{2}|x-y|^{2}\right)^{1+d / 2-p} \\
& \cdot M_{*}^{2} B_{6}^{*}\left(K^{2}+Q_{*}^{2}\left(\frac{\delta_{*}}{\ell}\right)^{2}\right) .
\end{aligned}
$$

Taking $p=d+2$ and integrating over $y$ we get this time, for some $B_{9}^{*}$ :

$$
\begin{aligned}
W_{t, s}^{2} & \leq\left(B_{9}^{*}\right)^{2} \delta_{*}^{d} k_{*}^{-d} k_{*}^{2 d} M_{*}^{2}\left(K+Q_{*} \frac{\delta_{*}}{\ell}\right)^{2} \\
& =\left(B_{9}^{*}\right)^{2} M_{*}^{2}\left(\delta_{*} k_{*}\right)^{d}\left(K+Q_{*} \frac{\delta_{*}}{\ell}\right)^{2} .
\end{aligned}
$$

Taking the square root of this bound, integrating over $s$, and noting that $\nu_{*} k_{*}^{2} \tau \leq 1$, we get

$$
\begin{aligned}
\int_{0}^{\tau} \mathrm{d} s\left|W_{\tau, s}\right| & \leq \int_{0}^{\tau} \mathrm{d} s B_{9}^{*} M_{*}\left(\delta_{*} k_{*}\right)^{d / 2}\left(K+Q_{*} \frac{\delta_{*}}{\ell}\right) \\
& =B_{9}^{*}\left(\frac{D_{*}}{\nu_{*}}\right)^{d / 4}\left(\frac{M_{*}}{\nu_{*} k_{*}^{2}}\right)^{1-d / 4}\left(K+Q_{*} \frac{\delta_{*}}{\ell}\right) .
\end{aligned}
$$


This completes the bound for $\nu_{*} k_{*}^{2} \tau \leq 1$.

The contribution from $w_{1,>}^{(1)}$ is easier to bound. Using the definition of $w_{1,>}^{(1)}$, we split the convolution integral into the region $|y| \leq \lambda$ and its complement. Because $\nu_{*} \tau_{*} \geq k_{*}^{-2}$ by Eq.(5.6) we can use the bound of Eq.(4.8), and we get for the first contribution when $p>d+1$,

$$
B_{3}^{*}(p)\left|\int_{|y| \leq \lambda} \mathrm{d} y \frac{e^{-\nu_{*} k_{*}^{2} \tau_{*} / 2}}{\left(\nu_{*} \tau_{*}\right)^{d / 2}\left(1+\frac{|x-y|^{2}}{D_{*} \tau_{*}}\right)^{p / 2}} w(y, 0)\right| \leq B_{10}^{*} e^{-\nu_{*} k_{*}^{2} \tau_{*} / 2} \sup _{|y| \leq \lambda}|w(y, 0)| .
$$

For the second term, where $|y| \geq \lambda$, the restriction of the bound to $|x| \leq \lambda$ - implies $|x-y| \geq \ell$, and then we get using $\tau_{*}=1 / M_{*}$ :

$$
\begin{aligned}
B_{3}^{*}(p) \mid & \int_{|x-y| \geq \ell} \mathrm{d} y \frac{e^{-\nu_{*} k_{*}^{2} \tau_{*} / 2}}{\left(\nu_{*} \tau_{*}\right)^{d / 2}\left(1+\frac{|x-y|^{2}}{D_{*} \tau_{*}}\right)^{p / 2}} w(y, 0) \mid \\
& \leq B_{11}^{*} \frac{\left(D_{*} \tau_{*}\right)^{1 / 2}}{\ell}\left(D_{*} \tau_{*}\right)^{d / 2} \frac{e^{-\nu_{*} k_{*}^{2} \tau_{*} / 2}}{\left(\nu_{*} \tau_{*}\right)^{d / 2}} \sup _{y}|w(y, 0)| \\
& =B_{11}^{*} \frac{\delta_{*}}{\ell}\left(\frac{D_{*}}{\nu_{*}}\right)^{d / 2} e^{-\nu_{*} k_{*}^{2} \tau_{*} / 2} Q_{*} .
\end{aligned}
$$

Combining Eqs.(5.13)-(5.17), the inequality (5.9) follows.

The proof of Eq.(5.8) is very similar to the one given above, and we indicate just the few modifications needed. Instead of the kernel $\mathcal{G}_{\tau,>}$ we now use the kernel $\mathcal{G}_{\tau}$, and we will call $Z_{t, s}$ the quantity corresponding to $Y_{t, s}$ but with $\mathcal{G}_{\tau}$ replaced by $\mathcal{G}_{\tau,>}$. Consider first $\nu_{*} \tau>1 / k_{*}^{2}$. Since the bound (4.8) is of the same type as the bound (4.1), but without he exponential factor, all bounds go through as before up to the inequality (5.12), which is replaced by a similar one, but without the exponential factor. Taking again the square root and integrating over $s$, we get for $d \leq 3$,

$$
\begin{aligned}
\left|w^{(2)}(x, \tau)\right| & \leq \int_{0}^{\tau} \mathrm{d} s\left|Z_{\tau, s}\right| \leq \int_{0}^{\tau_{*}} \mathrm{~d} s\left(B_{7}^{*}\right)^{2} \\
& \cdot M_{*}^{1-d / 4}\left(\nu_{*} k_{*}^{2}\right)^{d / 4}\left(K+Q_{*} \frac{\delta_{*}}{\ell}\right) \frac{1}{\left(\nu_{*} k_{*}^{2} s\right)^{d / 4}} \\
& \leq B_{12}^{*} M_{*}^{1-d / 4}\left(K+Q_{*} \frac{\delta_{*}}{\ell}\right) \tau_{*}^{1-d / 4} \\
& =B_{12}^{*}\left(K+Q_{*} \frac{\delta_{*}}{\ell}\right)
\end{aligned}
$$

This bounds the contribution of $w_{1}^{(2)}$ to Eq.(5.8) where $\nu_{*} \tau>1 / k_{*}^{2}$. In the opposite case, we argue exactly as in the proof of (5.9), since the exponential factor in (4.9) was anyway of no use before. Finally, the contribution of $w_{1}^{(1)}$ is bounded in exactly the same way as the one of $w_{1,>}^{(1)}$, except for the exponential factor, and we get the bound (5.8). The proof of Theorem 5.1 is complete. 


\section{The Sampling}

We assume that $w_{1}=w\left(\cdot, t=\tau_{*}\right)$ satisfies the "sampling bound"

$$
\left|w_{1}\right|_{\delta_{*}, \lambda} \leq \varepsilon
$$

where

$$
|f|_{\delta_{*}, \lambda} \equiv \sup _{\left\{n \in \mathbf{Z}^{d}: \delta_{*}|n| \leq \lambda\right\}}\left|f\left(n \delta_{*}\right)\right|
$$

with $\varepsilon \leq Q_{*}$. Furthermore, we assume

$$
\sup _{|x| \leq \lambda}|w(x, 0)| \leq K
$$

Then we have the

Theorem 6.1. Consider the solutions $w(\cdot, t)$ of Eq.(5.1), and assume that the bounds (5.2), (5.3) hold. There are constants $B_{13}^{*}, B_{14}^{*}$, and $B_{15}^{*}$ independent of $\varepsilon, K$, and $\lambda$ such that the following holds: If the initial condition satisfies (6.3) and $w\left(\cdot, \tau_{*}\right)$ satisfies Eq.(6.1) with the definition Eq.(5.5) of $\delta_{*}$, then

$$
\sup _{|x| \leq \lambda^{\prime}}\left|w\left(x, \tau_{*}\right)\right| \leq K^{\prime}
$$

where

$$
K^{\prime}=B_{13}^{*} \varepsilon+3 K / 4,
$$

and $\lambda^{\prime}=\lambda-B_{14}^{*}-B_{15}^{*} / K$.

Remark. Since $3 / 4<1$, Eq.(6.4) shows that $K^{\prime}$ is smaller than $K$ as long as $K>4 B_{13}^{*} \varepsilon$. (In fact, by choosing different constants-in particular a large $k_{*}$ - we can achieve any ratio $\rho>0$ instead of the $3 / 4$.)

A corollary of the proof is the following result which can be viewed as a generalization of the dissipative bounds of Theorem 5.1.

Corollary 6.2. In dimension $d \leq 3$ we have the following bounds

$$
\begin{aligned}
\sup _{|x| \leq \lambda-\ell}\left|w\left(x, \tau_{*}\right)\right| & \leq B_{16}^{*}\left|w\left(\cdot, \tau_{*}\right)\right|_{\delta_{*}, \lambda}+\frac{3}{4} \sup _{|x| \leq \lambda}|w(x, 0)| \\
& +B_{17}^{*} \frac{\delta_{*}}{\ell}\|w(\cdot, 0)\|_{\infty} .
\end{aligned}
$$

Proof. Our proof is based on sampling theory for functions in the Bernstein classes, (see, e.g., [B]).

Definition. We call $\mathcal{B}_{S, \sigma}$ the Bernstein class of entire analytic functions $f$ bounded by

$$
|f(z)| \leq S e^{\sigma|\operatorname{Im} z|}
$$


Our first observation is that $\mathcal{G}_{\tau,<}$ maps into a Bernstein class $\mathcal{B}_{B, 2 k_{*}}$ :

Lemma 6.3. The convolution operator with kernel

$$
\mathcal{G}_{\tau,<}(x-y)=\int \mathrm{d} k e^{i k(x-y)} e^{-D k^{2} \tau} \chi_{k<k_{*}}
$$

is bounded from $\mathrm{L}^{\infty}$ to itself. Furthermore, for $\tau \leq \tau_{*}$, it is bounded from $\mathrm{L}^{\infty}$ to the Bernstein class $\mathcal{B}_{B_{18}^{*}, 2 k_{*}}$, and $\mathcal{G}_{\tau,<} \star f$ is an analytic function of $z$ and one has the bound in $\mathbf{C}^{d}$ :

$$
\left|\left(\mathcal{G}_{\tau,<} \star f\right)(z)\right| \leq B_{18}^{*} e^{2 k_{*}|\operatorname{Im} z|}\|f\|_{\infty}
$$

Proof. This follows at once by taking $\mathrm{p}=\mathrm{d}+2$ in Lemma 4.2 and integrating.

Using this result, we can now bound the low-frequency part $w_{1,<}$ of $w_{1}(x)=w\left(x, \tau_{*}\right)$ :

Lemma 6.4. There is a constant $B_{19}^{*}$ such that the term $w_{1,<}$ is bounded by

$$
\left\|w_{1,<}(z)\right\|_{\infty} \leq B_{20}^{*} e^{2 k_{*}|\operatorname{Im} z|}\|w(\cdot, 0)\|_{\infty} \leq B_{19}^{*} e^{2 k_{*}|\operatorname{Im} z|}
$$

Proof. We recall the representation

$$
w_{1,<}(\cdot)=w_{<}\left(\cdot, \tau_{*}\right)=\mathcal{G}_{\tau_{*},<} \star w_{0}+\int_{0}^{\tau_{*}} \mathcal{G}_{\tau_{*}-s,<} \star(M(\cdot, s) w(\cdot, s)) .
$$

By Lemma 6.3, this is bounded as follows:

$$
\left|w_{<}\left(z, \tau_{*}\right)\right| \leq e^{2 k_{*}|\operatorname{Im} z|}\left(B_{18}^{*} Q_{*}+B_{2}^{*}(p) \tau_{*} M_{*} Q_{*}\right) \equiv e^{2 k_{*}|\operatorname{Im} z|} B_{19}^{*},
$$

so that Eq.(6.7) is proved.

Now that we have established that the function $w_{1,<}$ is entire analytic and exponentially bounded, we can use the following sampling result (written for functions in $d=1$ ):

Theorem 6.5. [B] Assume $f \in \mathcal{B}_{S, \sigma}$. The following representations hold:

$$
\begin{aligned}
\frac{f^{\prime}(0)}{\sigma} & =\frac{4 \sigma}{\pi^{2}} \sum_{n=-\infty}^{\infty} \frac{(-1)^{n}}{(2 n+1)^{2}} f\left(x_{2 n+1}\right) \\
f(x) & =f^{\prime}(0) \sin (\sigma x)+f(0) \frac{\sin (\sigma x)}{\sigma x}+\sigma x \sin (\sigma x) \sum_{n \neq 0} \frac{(-1)^{n}}{n \pi(\sigma x-n \pi)} f\left(x_{2 n}\right) .
\end{aligned}
$$

Here, $x_{n}=\frac{n \pi}{2 \sigma}$.

Remark. In higher dimensions, the sum is over a lattice, and details are left to the reader. 
Proof. [B]: Theorem 11.5.10 and Eq.(11.3.1). (There is an obvious dimensional misprint in 11.5.10.)

Note that the sums in Eqs.(6.8) and (6.9) are absolutely convergent. We now bound them as follows. Let $N$ be a (large) integer* Then, in the first expression, the sum over the terms with $|n|>N$ is bounded by $\mathcal{O}\left(N^{-1}\right)\|f\|_{\infty}$. In the second expression, we assume for the moment that $x \in\left(x_{0}, x_{2}\right)$. Then the sum over $|n|>N$ is bounded again by $\mathcal{O}\left(N^{-1}\right)\|f\|_{\infty}$.

Proposition 6.6. Assume that $f \in \mathcal{B}_{S, \sigma}$. Assume furthermore that $f$ satisfies the bounds

$$
\left|f\left(x_{j}\right)\right|<\alpha \text { for }|j| \leq J
$$

where $x_{j}=\pi j /(2 \sigma)$. Assume furthermore that $\|f\|_{\infty} \leq S^{\prime}$. Then, one has the bound

$$
|f(x)| \leq C_{6} \alpha+\frac{C_{7} S^{\prime}}{N} \text { for all }|x| \leq \pi(J-N-1) /(2 \sigma)
$$

The constants $C_{6}$ and $C_{7}$ are independent of the parameters of the problem. We shall assume $C_{6}>1$.

Proof. We first bound $f^{\prime}(0)$ using Eq.(6.8), and this leads to

$$
\sigma^{-1}\left|f^{\prime}(0)\right| \leq \frac{4}{\pi^{2}} \alpha \sum_{|n|<N} \frac{1}{(2 n+1)^{2}}+\frac{4}{\pi^{2}} S^{\prime} \sum_{|n| \geq N} \frac{1}{(2 n+1)^{2}} .
$$

If $|x| \leq J \pi /(2 \sigma)$, then $x \in\left(x_{2 \hat{n}}, x_{2 \hat{n}+2}\right)$ where $\hat{n}$ is the integer part of $\sigma x / \pi$. Note that by assumption we know that $\left|f\left(x_{2 n}\right)\right|<\alpha$ for all $n$ satisfying $|n-\hat{n}|<N$. Thus, splitting the sum as before and shifting the origin to $\hat{n} \pi / \sigma$, we get a bound

$$
\begin{aligned}
|f(x-(\hat{n} \pi / \sigma))| & \leq \frac{4}{\pi^{2}} \alpha \sum_{|n|<N} \frac{1}{(2 n+1)^{2}}+\frac{4}{\pi^{2}} S^{\prime} \sum_{|n| \geq N} \frac{1}{(2 n+1)^{2}} \\
& +\alpha+\alpha \sum_{|n|<N}\left|\frac{\sigma x \sin (\sigma x)}{n \pi(\sigma x-n \pi)}\right|+S^{\prime} \sum_{|n| \geq N} \frac{1}{2 \pi^{2} n^{2}}
\end{aligned}
$$

The assertion follows.

We can complete now the proof of Theorem 6.1. We first bound $w_{1,>}$. Setting $\lambda^{\prime \prime}=\lambda-\ell$ and using the bound Eq.(5.9), we get

$$
\begin{aligned}
\sup _{|x| \leq \lambda^{\prime \prime}}\left|w_{1,>}(x)\right| & \leq B_{5}^{*}\left(e^{-\left(\nu_{*} k_{*}^{2} / M_{*}\right) / 2}+\left(\frac{M_{*}}{\nu_{*} k_{*}^{2}}\right)^{1-d / 4}\right) \\
\cdot & \left(\sup _{|x| \leq \lambda}|w(x, 0)|+\left(\delta_{*} / \ell\right)\|w(\cdot, 0)\|_{\infty}\right) .
\end{aligned}
$$

\footnotetext{
* In this section, $N$ denotes just an integer, and not the number of components of $u$.
} 
We now begin fixing the constants: Recall that $Q_{*}, M_{*}$ and $\delta_{*}=D_{*} \tau_{*}$ are given by the parameters of the problem. We have also assumed, as a hypothesis of Theorem 6.1, that

$$
\sup _{|x| \leq \lambda}|w(x, 0)| \leq K .
$$

We choose $\ell=\delta_{*} Q_{*} / K$, so that the last factor in (6.10) is bounded by $2 K$. We next choose $k_{*}$ so large that

$$
B_{5}^{*}\left(e^{-\left(\nu_{*} k_{*}^{2} / M_{*}\right) / 2}+\left(\frac{M_{*}}{\nu_{*} k_{*}^{2}}\right)^{1-d / 4}\right) \leq \frac{1}{8 C_{6}} .
$$

More precisely, we let

$$
k_{*}=C_{8} 16 C_{6}\left(B_{5}^{*}\right)^{1 /(2-d / 2)}\left(M_{*} / \nu_{*}\right)^{1 / 2} \equiv B_{21}^{*}\left(M_{*} / \nu_{*}\right)^{1 / 2},
$$

and this is our final choice for $k_{*}$. Clearly, if $C_{8}$ is sufficiently large, both terms in the sum (6.11) will contribute less than $1 /\left(16 C_{6}\right)$.

Remark. It is at this point crucial that the construction of the quantities $B_{0}^{*}, B_{1}^{*}, \ldots, \operatorname{did}$ not depend on $k_{*}$, since all these constants depend — as we have said before — only on the quotient $D_{*} / \nu_{*}$. In particular, for problems where $u$ had only one component, this would mean that the $B_{j}^{*}$ are just pure numerical factors, since then $D_{*}=\nu_{*}$. It is also important to note that $k_{*}$ and $\delta_{*}$ do not depend on the quantities $\lambda$ or $K$ which occur in Theorem 6.1.

Thus, so far, with our choices we conclude from Eq.(6.10) that

$$
\sup _{|x| \leq \lambda^{\prime \prime}}\left|w_{1,>}(x)\right| \leq \frac{1}{4 C_{6}} K .
$$

We next observe that $w_{1,<}=w_{1}-w_{1,>}$ and so (6.13) leads to

$$
\left|w_{1,<}\left(n \delta_{*}\right)\right| \leq\left|w_{1}\left(n \delta_{*}\right)\right|+\left|w_{1,>}\left(n \delta_{*}\right)\right| \leq\left|w_{1}\left(n \delta_{*}\right)\right|+\frac{1}{4 C_{6}} K,
$$

provided $\delta_{*}|n| \leq \lambda^{\prime \prime}$

By construction, $w_{1,<}$ has a Fourier transform with support in $|k| \leq 2 k_{*}$ and furthermore, by Lemma 6.4, it is in $\mathcal{B}_{S, \sigma}$ with $S=B_{19}^{*}$ and $\sigma=2 k_{*}$.

We can now apply Proposition 6.6 to the function $f=w_{1,<}$, with $S^{\prime}=S=B_{19}^{*}$. Choosing $J=\left[2 \sigma \lambda^{\prime \prime} / \pi\right]$ (here, $[\cdot]$ is the integer part) we conclude that, for $|x| \leq \lambda^{\prime} \equiv$ $\lambda^{\prime \prime}-\delta_{*}-\pi(N+1) /\left(2 k_{*}\right)$, one has a bound

$$
\left|w_{1,<}(x)\right| \leq C_{6}\left|w_{1,<}\right|_{\delta_{*}, \lambda^{\prime \prime}}+C_{7} N^{-1} B_{19}^{*} .
$$

It is useful to introduce $B_{22}^{*}=C_{7} B_{19}^{*}$ Using Eq.(6.14), this leads to

$$
\sup _{|x| \leq \lambda^{\prime}}\left|w_{1,<}(x)\right| \leq C_{6}\left(\left|w_{1}\right|_{\delta_{*}, \lambda^{\prime \prime}}+\frac{1}{4 C_{6}} K\right)+N^{-1} B_{22}^{*} .
$$


We next choose

$$
N=\frac{1}{K} 4 B_{22}^{*}
$$

Then we get

$$
\sup _{|x| \leq \lambda^{\prime}}\left|w_{1,<}(x)\right| \leq C_{6}\left|w_{1}\right|_{\delta_{*}, \lambda^{\prime \prime}}+K / 2 .
$$

Combining Eqs.(6.18) and (6.13), we get

$$
\sup _{|x| \leq \lambda^{\prime}}\left|w_{1}(x)\right| \leq \sup _{|x| \leq \lambda^{\prime}}\left|w_{1,<}(x)\right|+\sup _{|x| \leq \lambda^{\prime \prime}}\left|w_{1,>}(x)\right| \leq C_{6}\left|w_{1}\right|_{\delta_{*}, \lambda^{\prime}}+\frac{K}{2}+\frac{K}{4 C_{6}} .
$$

Since we have taken $C_{6}>1$, we see that we get finally

$$
\sup _{|x| \leq \lambda^{\prime}}\left|w_{1}(x)\right| \leq C_{6}\left|w_{1}\right|_{\delta_{*}, \lambda^{\prime}}+3 K / 4,
$$

and

$$
\begin{aligned}
\lambda^{\prime} & =\lambda^{\prime \prime}-\delta_{*}-\pi(N+1) /\left(2 k_{*}\right) \\
& =\lambda-\delta_{*} B_{22}^{*} / K-\delta_{*}-\pi(N+1) /\left(2 k_{*}\right) .
\end{aligned}
$$

From the definition of $N=\mathcal{O}(1 / K)$ it follows at once that there are constants $B_{14}^{*}$ and $B_{15}^{*}$ such that

$$
\lambda^{\prime} \geq \lambda-B_{14}^{*}-B_{15}^{*} / K .
$$

The proof of Theorem 6.1 is complete.

Theorem 6.7. (Sampling bound) Consider the solutions $w(\cdot, t)$ of Eq.(5.1), and assume that the bounds (5.2), (5.3) hold. There are constants $B_{23}^{*}, B_{24}^{*}$ and $B_{25}^{*}$ such that if

$$
\left|w\left(i \delta_{*}, t-j \tau_{*}\right)\right| \leq \varepsilon,
$$

for all $|i| \leq\left(L+B_{23}^{*} / \varepsilon\right) / \delta_{*}$ and all $|j| \leq B_{24}^{*} \log (1 / \varepsilon)$, then

$$
\sup _{|x| \leq L}|w(x, t)| \leq B_{25}^{*} \varepsilon .
$$

Remark. It will be seen from the proof that it suffices to sample on a somewhat smaller, non-rectangular domain for (6.20) to hold. See also Fig. 1.

Proof. We obtain the result of Theorem 6.7 by iteration of Theorem 6.1. With $\varepsilon$ given as in the statement of Theorem 6.7, let $m$ be the smallest integer for which

$$
(3 / 4)^{m} Q_{*} \leq \varepsilon
$$

and note that then

$$
m \leq \log \left(Q_{*} / \varepsilon\right) / \log (4 / 3)+1 \leq C_{9} \log (1 / \varepsilon),
$$


when $\varepsilon<\frac{1}{2}$. The number $m$ will be the number of time steps needed to achieve a precision $B_{25}^{*} \varepsilon$ in Eq.(6.20), where we define $B_{25}^{*}=2 B_{13}^{*}$, and $B_{24}^{*}=C_{9}$. Let now $K_{0}=Q_{*}$ and $K_{j+1}=B_{13}^{*} \varepsilon+3 K_{j} / 4$, and let $L_{m}=L$, and $L_{j-1}=L_{j}+\delta_{*}+B_{15}^{*} / K_{j-1}$. Assume now

$$
\left|w\left(i \delta_{*}, t-j \tau_{*}\right)\right| \leq \varepsilon,
$$

for all $|i| \leq L_{m-i} / \delta_{*}$ and for all $j=0, \ldots, m$. The reader can check easily that our definitions are made such that Theorem 6.1 applies at each time step considered. Since we have

$$
\left\|w\left(\cdot, t-j \tau_{*}\right)\right\|_{\infty} \leq Q_{*},
$$

for all $j$, we can inductively bound, for $j=1, \ldots, m$,

$$
\sup _{|x| \leq L_{j}}\left|w\left(x, t-(m-j) \tau_{*}\right)\right| \leq C_{6} \varepsilon+3 K_{j-1} / 4=K_{j+1} .
$$

Note now that $K_{j}=Q_{*} \rho^{j}+C_{6} \varepsilon\left(1-\rho^{j}\right) /(1-\rho)$, where $\rho=3 / 4$ and thus

$$
L_{m-j}=L+j \delta_{*}+B_{15}^{*} \sum_{i=1}^{j} K_{m-i}^{-1} .
$$

Clearly, there is a $C_{10}>0$ such that $K_{j} \geq C_{10} \rho^{j}$, so that we get

$$
L_{m-j}=L+j \delta_{*}+\frac{B_{15}^{*}}{C_{10}} \sum_{i=1}^{j} \frac{1}{\rho^{m-j}} .
$$

In particular, we can find some $C_{11}$, so that

$$
L_{0} \leq L+m \delta_{*}+\frac{B_{15}^{*}}{C_{10}} \frac{\rho^{-m}}{\rho^{-1}-1} \leq L+C_{9} \delta_{*} \log (1 / \varepsilon)+C_{11} / \varepsilon .
$$

Note that $L_{0}$ is the width of the "earliest" bound in (6.19), and we choose $B_{23}^{*}=C_{9} \delta_{*}+C_{11}$. Since $K_{m} \leq B_{13}^{*} \varepsilon$ we have shown that the bounds of Eq.(6.21) are sufficient to ensure

$$
\sup _{|x| \leq L}|w(x, t)| \leq 2 B_{13}^{*} \varepsilon .
$$

The proof of Theorem 6.7 is completed. 


\section{Outlook}

In this section, we wish to discuss potential experimental aspects of our results. These aspects must necessarily rely on a number of conjectures about the system under consideration, and are similar in spirit to the discussion found in [ER]. While we have defined topological entropy in Section 2, we now need to address the question of entropy relative to an invariant measure $\mu$. We shall call it $h_{\mu}$. More precisely, let $f$ be a continuous map of a compact metric space and let $\mu$ be an $f$-invariant non-atomic ergodic measure. The entropy $h_{\mu}(f)$ is determined as follows. For $\varepsilon>0$ and an integer $n>0$ let

$$
V(x, \varepsilon, n)=\left\{y \mid d\left(f^{i}(y), f^{i}(x)\right)<\varepsilon, 0 \leq i<n\right\} .
$$

One has (see $[\mathrm{BK}],[\mathrm{Y}])$ :

$$
h_{\mu}(f)=-\lim _{\varepsilon \rightarrow 0} \liminf _{n \rightarrow \infty} \frac{1}{n} \log \mu(V(x, \varepsilon, n)) .
$$

This is not a very convenient expression for explicit computations. As for the case of finite dimension (see [GP]) one can try to determine instead a correlation entropy. In our case we would also like to include the dependence on the size of the window in which the system is observed. One is naturally lead to the following definition of correlation entropy per unit length $K_{2}(\mu)$-and unit time-(see [GP, ER]) for a measure $\mu$ which is space and time invariant and ergodic. The definition of this quantity is:

$$
\begin{aligned}
K_{2}(\mu)= & -\frac{1}{\tau} \lim _{\varepsilon \rightarrow 0} \lim _{L \rightarrow \infty} \frac{1}{L^{d}} \lim _{n \rightarrow \infty} \frac{1}{n} \\
& \log \left(\lim _{N \rightarrow \infty} \frac{1}{N^{2}} \sum_{j, k=0}^{N-1} \prod_{i=0}^{n-1} \Theta\left(\varepsilon-\left\|\Phi_{(i+j) \tau}(A)-\Phi_{(i+k) \tau}(A)\right\|_{L^{\infty}\left(Q_{L}\right)}\right)\right),
\end{aligned}
$$

where $\Theta$ is the Heaviside function, and $\Phi_{t}$ is the flow of the dynamics.

It is an open question to prove that the limits in the above expression exist for $\mu$ almost every $A$ (except the limit over $\varepsilon$ since the quantity is increasing). Furthermore, the result should also be independent of the time step $\tau$. But let us assume that these limits exist. In that case our sampling bound Theorem 6.7 gives us a constructive handle on computing the r.h.s of Eq.(7.1). We recall the definition

$$
|f|_{\delta, \lambda} \equiv \sup _{\left\{n \in \mathbf{Z}^{d}: \delta|n| \leq \lambda\right\}}|f(n \delta)|
$$

Theorem 7.1. Assuming the limits in (7.1) exist for $\mu$-almost every $A$, we have for every $\delta \in\left(0, \delta_{*}\right]$ and every $\tau \in\left(0, \tau_{*}\right]$ :

$$
\begin{aligned}
K_{2}(\mu)= & -\frac{1}{\tau} \lim _{\varepsilon \rightarrow 0} \lim _{L \rightarrow \infty} \frac{1}{L^{d}} \lim _{n \rightarrow \infty} \frac{1}{n} \\
& \log \left(\lim _{N \rightarrow \infty} \frac{1}{N^{2}} \sum_{j, k=0}^{N-1} \prod_{i=0}^{n-1} \Theta\left(\varepsilon-\left|\Phi_{(i+j) \tau}(A)-\Phi_{(i+k) \tau}(A)\right|_{\delta, L}\right)\right) .
\end{aligned}
$$


Remark. Note that the sum $\sum_{j, k=0}^{N-1} \prod_{i=0}^{n-1} \Theta(\ldots)$ counts the number of pairs of points in the sample which have a "distance" of less than $\varepsilon$ in embedding dimension $n$, where the distance is measured with the discrete sampling step $\delta$ over a region $|x| \leq L$. In this sense, our result says that for PDE's on the infinite line, measuring the $K_{2}$ entropy can be done by the usual Grassberger-Procaccia algorithm [GP, ER].

Remark. If we choose $\delta=\delta_{*}$ and $\tau=\tau_{*}$, then the "sampling error" is controlled by the constants of the proof of Theorem 6.7. But if we choose, for example $\tau=\tau_{*} / 2$, the number of time steps-i.e., the factor $B_{24}^{*}$-needed in Theorem 6.7 will be the same as if $M_{*}$ had been replaced by $2 M_{*}$ in the original assumptions. So clearly, the total time during which one must measure to achieve a given sampling precision can not be shortened by sampling at shorter intervals. Similar precautions are necessary for sampling in space.

Proof. In view of the remark, we give the proof only for the case $\tau=\tau_{*}, \delta=\delta_{*}$, in which case the constants $B_{23}^{*}, \ldots$, retain their meaning from earlier parts of the paper. We obviously have

$$
\begin{gathered}
\frac{1}{N^{2}} \sum_{j, k=0}^{N-1} \prod_{i=0}^{n-1} \Theta\left(\varepsilon-\left|\Phi_{(i+j) \tau_{*}}(A)-\Phi_{(i+k) \tau_{*}}(A)\right|_{\delta_{*}, L}\right) \\
\geq \frac{1}{N^{2}} \sum_{j, k=0}^{N-1} \prod_{i=0}^{n-1} \Theta\left(\varepsilon-\left\|\Phi_{(i+j) \tau_{*}}(A)-\Phi_{(i+k) \tau_{*}}(A)\right\|_{L^{\infty}\left(Q_{L}\right)}\right) .
\end{gathered}
$$

On the other hand, applying Theorem 6.7, we have for $n>B_{24}^{*} \log (1 / \varepsilon)+1$ and $L>B_{23}^{*} / \varepsilon$ :

$$
\begin{array}{r}
\prod_{i=0}^{n-1} \Theta\left(\varepsilon-\left|\Phi_{(i+j) \tau_{*}}(A)-\Phi_{(i+k) \tau_{*}}(A)\right|_{\delta_{*}, L}\right) \\
\leq \prod_{i=\left[B_{24}^{*} \log (1 / \varepsilon)\right]}^{n-1} \Theta\left(B_{25}^{*} \varepsilon-\left\|\Phi_{(i+j) \tau_{*}}(A)-\Phi_{(i+k) \tau_{*}}(A)\right\|_{L^{\infty}\left(Q_{L-B_{23}^{*} / \varepsilon}\right)}\right) \\
\leq \prod_{i=0}^{n-1-\left[B_{24}^{*} \log (1 / \varepsilon)\right]} \Theta\left(B_{25}^{*} \varepsilon-\| \Phi_{\left(i+j+\left[B_{24}^{*} \log (1 / \varepsilon)\right]\right) \tau_{*}}(A)\right. \\
\left.-\Phi_{\left(i+k+\left[B_{24}^{*} \log (1 / \varepsilon)\right]\right) \tau_{*}}(A) \|_{L^{\infty}\left(Q_{L-B_{23}^{*} / \varepsilon}\right)}\right) .
\end{array}
$$

Therefore,

$$
\begin{aligned}
& \frac{1}{N^{2}} \sum_{j, k=0}^{N-1} \prod_{i=0}^{n-1} \Theta\left(\varepsilon-\left|\Phi_{(i+j) \tau_{*}}(A)-\Phi_{(i+k) \tau_{*}}(A)\right|_{\delta_{*}, L}\right) \\
& \quad \leq \frac{1}{N^{2}} \sum_{j, k=0}^{N-1} \prod_{i=0}^{n-1-\left[B_{24}^{*} \log (1 / \varepsilon)\right]} \Theta\left(B_{25}^{*} \varepsilon-\left\|\Phi_{(i+j) \tau_{*}}(A)-\Phi_{(i+k) \tau_{*}}(A)\right\|_{L^{\infty}\left(Q_{L-B_{23}^{*} \varepsilon}\right)}\right) \\
& \quad+\frac{1}{N} \mathcal{O}(1+\log (1 / \varepsilon))^{2} .
\end{aligned}
$$


The result now follows by taking the limits $N \rightarrow \infty, n \rightarrow \infty, L \rightarrow \infty$ (in that order) for $\varepsilon$ fixed, and then letting $\varepsilon$ tend to zero.

\section{References}

[AKM] Adler, R., Konheim, A.G. and McAndrew, M.H.: Topological Entropy. Trans. Am. Math. Soc. 114, 390 (1965).

[B] Boas, R.P.: Entire Functions, New York: Academic Press (1954).

[BK] Brin, M. and Katok, A.: On local entropy. In Geometric Dynamics (Rio de Janeiro, 1981), Lecture Notes in Mathematics 1007. 30-38 (1983).

[CJT] Cockburn, B., Jones, D.A. and Titi, E.: Estimating the number of asymptotic degrees of freedom for nonlinear dissipative systems. Math. Comput. 66, 1073-1087 (1997).

[C] Collet, P.: Thermodynamic limit of the Ginzburg-Landau equation. Nonlinearity 7, 1175-1190 (1994).

[CE1] Collet, P. and Eckmann, J.-P.: The time-dependent amplitude equation for the Swift-Hohenberg problem. Commun. Math. Phys. 132, 139-153 (1990).

[CE2] Collet, P. and Eckmann, J.-P.: Extensive properties of the complex Ginzburg-Landau equation. Preprint. (1998).

[ER] Eckmann, J.-P. and Ruelle, D.: Ergodic theory of chaos and strange attractors. Rev. Mod. Phys. 57, 617-656 (1985).

[ER2] Eckmann, J.-P. and Ruelle, D.: Fundamental limitations for estimating dimensions and Liapunov exponents in dynamical systems. Physica D56, 185-187 (1992).

[GP] Grassberger, P. and I. Procaccia: Estimating the Kolmogorov entropy from a chaotic signal. Phys. Rev. A28, 2591 (1983).

[GV] Ginibre, J. and G. Velo: The Cauchy problem in local spaces for the complex Ginzburg-Landau equation. II: contraction methods. Commun. Math. Phys. 187, 45-79 (1997).

[KT] Kolmogorov, A.N. and Tikhomirov, V.M.: $\varepsilon$-entropy and $\varepsilon$-capacity of sets in functional spaces ${ }^{1}$. In Selected Works of A.N Kolmogorov, Vol III, (Shirayayev, A.N., ed.). Dordrecht, Kluver (1993).

[MS] Mielke, A. and Schneider, G.: Attractors for modulation equations on unbounded domains-existence and comparison. Nonlinearity 8, 743-768 (1995).

[Y] Young, L.S.: Dimension, entropy and Lyapunov exponents. Erg. Th. Dyn. Sys. 2, 109-124 (1982).

\footnotetext{
1 The version in this collection is more complete than the original paper of Uspekhi Mat. Nauk, 14, 3-86 (1959).
} 